MATHEMATICS OF COMPUTATION

Volume 74, Number 250, Pages 543-554

S 0025-5718(04)01711-9

Article electronically published on August 31, 2004

\title{
A NEW FAMILY OF STABLE MIXED FINITE ELEMENTS FOR THE 3D STOKES EQUATIONS
}

\author{
SHANGYOU ZHANG
}

\begin{abstract}
A natural mixed-element approach for the Stokes equations in the velocity-pressure formulation would approximate the velocity by continuous piecewise-polynomials and would approximate the pressure by discontinuous piecewise-polynomials of one degree lower. However, many such elements are unstable in $2 \mathrm{D}$ and $3 \mathrm{D}$. This paper is devoted to proving that the mixed finite elements of this $\mathbf{P}_{k}-P_{k-1}$ type when $k \geq 3$ satisfy the stability conditionthe Babuška-Brezzi inequality on macro-tetrahedra meshes where each big tetrahedron is subdivided into four subtetrahedra. This type of mesh simplifies the implementation since it has no restrictions on the initial mesh. The new element also suits the multigrid method.
\end{abstract}

\section{INTRODUCTION}

The analysis of mixed methods for the Stokes problem (cf. the books 2] and 4 for references) can be based on the general theory of saddle point problems where the central task is to verify an inf-sup stability condition - the Babuška-Brezzi inequality. However many mixed-element pairs fail to satisfy this inequality. In the velocity-pressure formulation of the Stokes equations, the velocity and pressure are in the Sobolev spaces $H^{1}(\Omega)^{d}$ and $L_{0}^{2}(\Omega)$, respectively. A natural approach of approximation would then be to use continuous piecewise-polynomials of degree $k$ or less for the velocity and discontinuous piecewise-polynomials of degree $(k-1)$ or less for the pressure. Such mixed element solutions satisfy the incompressibility condition, pointwise. Scott and Vogelius showed that the Babuška-Brezzi inequality holds for the natural mixed triangular elements in $2 \mathrm{D}$ if $k \geq 4$ and if the meshes are singular-vertex free (see [8]). An internal vertex in $2 \mathrm{D}$ is said to be singular if edges meeting at the point fall into two straight lines. The problem of finding such a minimal degree $k$ in 3D was posed by Scott and Vogelius in 8 almost two decades ago, but it remains open. The study in 3D is difficult since one has to search for all possible types of problematic vertices or edges, similar to the $2 \mathrm{D}$ singular vertex found in 8 . The $3 \mathrm{D}$ triangulation is complicated and would be even worse if additional constraints are applied to avoid singularities, if they are known. Furthermore, if one applies the multigrid method to solve such tetrahedral mixed elements, the multigrid refinement would produce a known-type singular edge; i.e., all triangles meeting at one edge fall into two planes, when refining each tetrahedron

Received by the editor October 3, 2002 and, in revised form, January 9, 2003.

2000 Mathematics Subject Classification. Primary 65N30, 65F10.

Key words and phrases. Stokes problem, finite element, mixed element, inf-sup condition, multigrid method. 
into eight half-sized tetrahedra. Some additional comments on computation with a singular vertex are presented at the end of this paper.

In this paper, we will extend the result of Scott and Vogelius in [8] in a different direction. Instead of finding all possible types of singular vertices and edges, we propose a new macro-element type mesh which is shown to have no singular vertices or singular edges; i.e., the space of divergence of $\mathbf{P}_{k}^{0}$ is equal to the space $P_{k-1}$ on such meshes. We will show that the $\mathbf{P}_{k}-P_{k-1}$ element is stable if the polynomial degree $k$ for velocity is 3 or higher. In the new method, starting with any quasiuniform tetrahedral mesh, the mesh for mixed-elements is generated by subdividing each initial tetrahedron into four subtetrahedra by connecting the bary-center with four vertices (see Figure 1). The $\mathbf{P}_{k}-P_{k-1}$ mixed elements are defined on this new mesh. The velocity of the mixed-element solution is divergence free pointwise. The significance of the new approach is in the two aspects mentioned above, i.e., reducing the difficulty in mesh generator and allowing the possibility of applying the multigrid method. When applying the multigrid algorithm to the new macroelements, we should remove the internal triangles first to turn the four subtetrahedra back into one element. We now refine each big tetrahedron into eight subtetrahedra as usual (note that the new subtetrahedra may not always be similar to the parent tetrahedron; see [13-14]) and then subdivide each of eight subtetrahedra into four subsubtetrahedra to get the grid on the next level. The multilevel meshes generated this way are not nested and nonnested multigrid features are needed here (cf. [15][16]).

The analysis for the stability of the macro-element type $\mathbf{P}_{k}-P_{k-1}$ mixed element is based on a framework of Stenberg [11]. The only work left to do is to show a macro-element condition (see (4) below). The same approach has been adopted by Qin in [5] where the quadratic and cubic mixed macro-elements in 2D are shown to be stable (the case $k \geq 4$ is proved for general meshes by [8]). Some low-order 3D mixed-elements on tetrahedral meshes have been studied in [1] and [12], but the finite element spaces for pressure are continuous there.

\section{Mixed finite Elements}

We consider the stationary Stokes problem: Find functions $\vec{\psi}$ (the fluid velocity) and $\tau$ (the pressure) on a 3D polyhedral domain $\Omega$ such that

$$
\begin{aligned}
-\nu \Delta \vec{\psi}+\nabla \tau=\mathbf{f} & \text { in } \Omega, \\
\operatorname{div} \vec{\psi}=0 & \text { in } \Omega, \\
\vec{\psi}=\mathbf{0} & \text { on } \partial \Omega,
\end{aligned}
$$

where $\mathbf{f}$ is the body force and $\nu$ is the kinematic viscosity. We adopt notation in 11] or conventional notation. Given a quasi-uniform (cf. 3]) tetrahedral mesh $\mathcal{M}_{h}=\{M\}$, where $h$ denotes the mesh size, on $\Omega$, we let $\mathcal{T}_{h}=\{T\}$ denote the corresponding macro-element mesh where each tetrahedron $M$ of $\mathcal{M}_{h}$ is cut into four by connecting the bary-center with four vertices as depicted in Figure 1. Let $\mathbf{P}_{k, h, \Omega}^{0}$ and $P_{k, h, \Omega}^{-1}$ denote the vector (three components) piecewise-continuous and piecewise-discontinuous polynomials of degree $k$ or less on the mesh $\mathcal{T}_{h}$, respectively. When there is no confusion, we will use shorthand notation like $\mathbf{P}_{k}$ for $\mathbf{P}_{k, h, \Omega}^{0}$, or $\mathbf{P}_{k, h, M}^{0}$, or $\mathbf{P}_{k, h_{0}, M_{0}}^{0}$ where $M$ is an element in $\mathcal{M}_{h}$ and $M_{0}$ is the reference, unit $\left(h_{0}=\sqrt{2}\right)$ macro-element depicted in Figure 1. Similarly, $P_{k-1}$ stands for $P_{k-1, h}^{-1}$. 


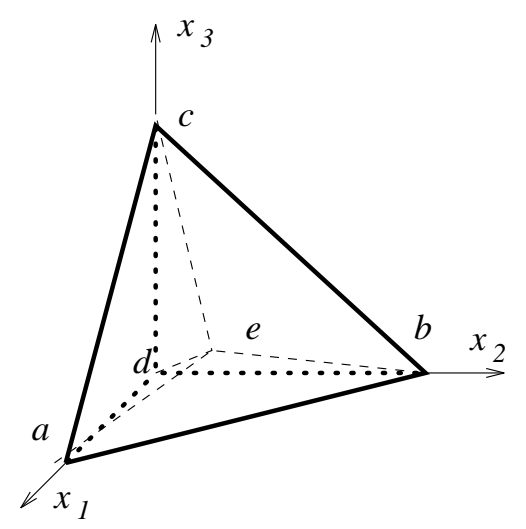

FiguRE 1. The reference macro-element $M_{0}$ (a unit tetrahedron).

It is understood that boldface letter $\mathbf{P}$ stands for a continuous piecewise-polynomial space while the letter $P$ stands for a discontinuous one. Let

$$
\begin{aligned}
& \stackrel{\circ}{\mathbf{P}}_{k}=\mathbf{P}_{k} \cap H_{0}^{1}(\Omega)=\left\{\mathbf{v} \in \mathbf{P}_{k, h, \Omega}^{0} \mid \mathbf{v}=0 \text { on } \partial \Omega\right\}, \\
& \stackrel{\circ}{P}_{k}=P_{k} \cap L_{0}^{2}(\Omega)=\left\{p \in P_{k, h, \Omega}^{-1} \mid \int_{\Omega} p=0\right\} .
\end{aligned}
$$

The mixed elements approximation to (1) in weak formulation is: Find $\mathbf{u}_{h} \in \stackrel{\circ}{\mathbf{P}}_{k}$ and $p_{h} \in \stackrel{\circ}{P}_{k-1}$ such that

$$
\begin{aligned}
\nu\left(\nabla \mathbf{u}_{h}, \nabla \mathbf{v}\right)-\left(\operatorname{div} \mathbf{v}, p_{h}\right) & =(\mathbf{f}, \mathbf{v}) & & \forall \mathbf{v} \in \stackrel{\circ}{\mathbf{P}}_{k}, \\
\left(\operatorname{div} \mathbf{u}_{h}, \mu\right) & =0 & & \forall \mu \in \stackrel{\circ}{P}_{k-1} .
\end{aligned}
$$

The Babuška-Brezzi stability condition reads: there exists a constant $C>0$ such that

$$
\sup _{\substack{\mathbf{v} \in \stackrel{\mathbf{P}}{*}_{k}, \mathbf{v} \neq \mathbf{0} \\ \mid}} \frac{(\operatorname{div} \mathbf{v}, p)}{|\mathbf{v}|_{1}} \geq C\|p\|_{0} \quad \forall p \in \stackrel{\circ}{P}_{k-1}
$$

which ensures the convergence of the mixed elements solutions. In [1], it is shown that the following "macro-element condition" is sufficient for (3) to be valid:

$$
\begin{aligned}
& \operatorname{dim}\left(N_{M}\right)=1 \quad \text { for all } M \in \mathcal{M}_{h}, \\
& \text { where } N_{M} \stackrel{\text { def }}{=}\left\{p \in P_{k-1, h, \Omega}^{-1} \mid(\operatorname{div} \mathbf{v}, p)=0 \forall \mathbf{v} \in{\stackrel{\circ}{\mathbf{P}_{k, h, M}}}_{k,}\right\}
\end{aligned}
$$

if $k \geq 3$. The condition $k \geq 3$ is only to guarantee that there is at least one degree of freedom for the discrete velocity functions in the interior of each face triangle of tetrahedron (cf. Lemma 3.3 in [1]). Under this condition, the analysis for the $2 \mathrm{D}$ case in [11] remains the same in $3 \mathrm{D}$. In particular, to be rigorous, we can use the polynomial interpolation result in [9] which defines precisely the interpolation operator needed in the proof of Lemma 3.3 in [11]. We now verify the condition (4), which only needs to be done on the reference macro-element.

Let $M_{0}$ be the unit reference tetrahedron $a b c d$ at the origin, as depicted in Figure 1. We denote the bary-center $\left(\frac{1}{4}, \frac{1}{4}, \frac{1}{4}\right)$ by $e$. To use bary-centric coordinates, we 
define the 4 th variable $x_{4}=1-x_{1}-x_{2}-x_{3}$. Sequentially, we denote the four subtetrahedra bcde, dcae, abde and acbe by $T_{1}, T_{2}, T_{3}$ and $T_{4}$. We note that the subtetrahedron $T_{i}$ has precisely one boundary triangle of $M_{0}$ as its face.

\section{ANALYSis}

In this section, we will show the perfect matching of the mixed finite element pairs on the reference macro-element $M_{0}: \operatorname{div}{\stackrel{\circ}{\mathbf{P}^{\prime}}}_{k, h_{0}, M_{0}}^{0}=\stackrel{\circ}{P}_{k-1, h_{0}, M_{0}}^{-1}$. It is to be done by mathematical induction. By this result, we will obtain the stability and the optimal order of convergence for the mixed finite elements by the framework of Stenberg [11].

Lemma 1. If $p \in \stackrel{\circ}{P}_{k-1}$ satisfies

$$
\int_{M_{0}} \operatorname{div} \mathbf{u} p d \mathbf{x}=0
$$

for all $\mathbf{u} \in \stackrel{\circ}{\mathbf{P}}_{1}$, then

$$
\int_{T_{i}} p d \mathbf{x}=0, \quad 1 \leq i \leq 4
$$

In particular, if $p$ is in $\stackrel{\circ}{P}_{0}$, then $p \equiv 0$ on $M_{0}$.

Proof. Let $v$ be the piecewise linear nodal basis function at $e$, i.e., $v(e)=1$ and $\left.v\right|_{\partial M_{0}}=0$. In fact, $\left.v\right|_{T_{i}}=4 x_{i}$ on each of four subtetrahedra. Defining $\mathbf{u}_{i}$ as the vector function whose $i$ th component is $v$ and the other two components are 0 . A simple calculation would lead to

$$
\operatorname{div}_{i}=\left\{\begin{array}{ll}
0 & \mathbf{x} \in T_{j}, 1 \leq j \leq 3, j \neq i, \\
4 & \mathbf{x} \in T_{i}, \\
-4 & \mathbf{x} \in T_{4},
\end{array} \quad \text { for } 1 \leq i \leq 3 .\right.
$$

Letting $\mathbf{u}=\mathbf{u}_{i}$ in (5), we get three equations. Now as $p \in \stackrel{\circ}{P}_{k-1}$ (the fourth equation), we get (6). If $p \in \stackrel{\circ}{P}_{0}$, then $p$ is a piecewise constant function and (6) would ensure that all four constants on the four subtetrahedra are the same, 0 .

We may not need the next two lemmas in order to prove Theorem 4, where Lemma 1 is needed in order to apply the method of mathematical induction. But for two reasons, we listed the proof of the next two lemmas. The first reason is to help readers understand nodal basis functions and the approach of the proof. The second reason is to shorten the proof of Theorem 4 .

Lemma 2. If $p \in \stackrel{\circ}{P}_{k-1}$ satisfies (5) for all $\mathbf{u} \in \stackrel{\circ}{\mathbf{P}}_{2}$, then

$$
\int_{T_{i}} x_{j} p d \mathbf{x}=0, \quad i, j=1,2,3,4 .
$$

In particular, if $p$ is in $\stackrel{\circ}{P}_{1}$, then $p \equiv 0$ on $M_{0}$. 
Proof. Let $v_{0}$ be the piecewise-quadratic nodal basis function at the bary-center $e$, i.e., $\left.v_{0}\right|_{T_{i}}=32 x_{i}\left(x_{i}-\frac{1}{8}\right)$. Constructing three vector functions by $v_{0}$ as we $\operatorname{did}$ before (7), we would get

$$
\int_{T_{i}} x_{i} p d \mathbf{x}=\int_{T_{j}} x_{j} p d \mathbf{x}, \quad i, j=1,2,3,4 .
$$

Let $v_{i}$ be the piecewise-quadratic nodal basis function at the midpoint

$$
\left(\frac{\delta_{1 i}}{2}+\frac{1}{8}, \frac{\delta_{2 i}}{2}+\frac{1}{8}, \frac{\delta_{3 i}}{2}+\frac{1}{8}\right)
$$

of one of the three interior edges, ae, be and $c e$ of $M_{0}$, where $\delta_{j i}$ is the Kronecker delta symbol. For example, we have

$$
v_{1}(\mathbf{x})= \begin{cases}0, & \mathbf{x} \in T_{1} \\ 16 x_{2}\left(x_{1}-x_{2}\right), & \mathbf{x} \in T_{2} \\ 16 x_{3}\left(x_{1}-x_{3}\right), & \mathbf{x} \in T_{3} \\ 16 x_{4}\left(x_{1}-x_{4}\right), & \mathbf{x} \in T_{4}\end{cases}
$$

$$
\operatorname{div}\left(\begin{array}{l}
v_{1} \\
0 \\
0
\end{array}\right)=\left\{\begin{array}{ll}
0 & \text { in } T_{1}, \\
16 x_{2} & \text { in } T_{2}, \\
16 x_{3} & \text { in } T_{3}, \\
16\left(3 x_{4}-x_{1}\right) & \text { in } T_{4},
\end{array} \text { and } \quad \operatorname{div}\left(\begin{array}{c}
0 \\
v_{1} \\
0
\end{array}\right)= \begin{cases}0 & \text { in } T_{1}, \\
16\left(x_{1}-2 x_{2}\right) & \text { in } T_{2}, \\
0 & \text { in } T_{3}, \\
16\left(2 x_{4}-x_{1}\right) & \text { in } T_{4} .\end{cases}\right.
$$

By (5), (9), (10) and the symmetry, it follows that

$$
\int_{T_{i}} x_{j} p d \mathbf{x}=5 \int_{T_{i}} x_{i} p d \mathbf{x}, \quad i, j=1,2,3,4 \text { and } i \neq j .
$$

In particular, on tetrahedron $T_{1}$, we have

$$
\begin{aligned}
& 5 \int_{T_{1}} x_{1} p d \mathbf{x}=\int_{T_{1}} x_{2} p d \mathbf{x}, \\
& 5 \int_{T_{1}} x_{1} p d \mathbf{x}=\int_{T_{1}} x_{3} p d \mathbf{x}, \\
& 5 \int_{T_{1}} x_{1} p d \mathbf{x}=\int_{T_{1}} x_{4} p d \mathbf{x} .
\end{aligned}
$$

Noting $x_{4}=1-x_{1}-x_{2}-x_{3}$, we can add (11) to get $16 \int_{T_{1}} x_{1} p d \mathbf{x}=\int_{T_{1}} p d \mathbf{x}=0$ where we applied (6). Therefore (8) is proved. For any $p \in \stackrel{\circ}{P}_{1}$, a linear combination of (8) would lead $\int_{M_{0}} p^{2} d \mathbf{x}=0$, which implies $p \equiv 0$.

Before we apply the principle of mathematical induction to prove the general case of arbitrary degree $k$, we repeat Lemmas 1 and 2 for $\stackrel{\circ}{\mathbf{P}}_{3}$.

Lemma 3. If $p \in \stackrel{\circ}{P}_{k-1}$ satisfies (5) for all $\mathbf{u} \in \stackrel{\circ}{\mathbf{P}}_{3}$, then

$$
\int_{T_{i}} x_{j} x_{k} p d \mathbf{x}=0, \quad i, j, k=1,2,3,4 .
$$

In particular, if $p$ is in $\stackrel{\circ}{P}_{2}$, then $p \equiv 0$ on $M_{0}$. 
Proof. Again, first let $v$ be the piecewise-cubic nodal basis function at the barycenter: $\left.v_{3,0}\right|_{T_{i}}=288 x_{i}\left(x_{i}-\frac{1}{12}\right)\left(x_{i}-\frac{1}{6}\right)$. Letting $\mathbf{u}=\left(v_{3,0} 00\right)^{\top}$ in (5), we would get $\int_{T_{1}} x_{1}^{2} p d \mathbf{x}=\int_{T_{4}} x_{4}^{2} p d \mathbf{x}$. By symmetries, all values of $\int_{T_{i}} x_{i}^{2} p d \mathbf{x}$ are the same:

$$
\int_{T_{i}} x_{i}^{2} p d \mathbf{x}=\int_{T_{j}} x_{j}^{2} p d \mathbf{x}, \quad 1 \leq i, j \leq 4
$$

Here we used the identities listed in (6) and (8) for lower degree polynomials. Next let $v_{3,1}$ be the nodal basis function at the node with coordinates $(a+2 e) / 3$ :

$$
v_{3,1}(\mathbf{x})= \begin{cases}0, & \mathbf{x} \in T_{1}, \\ 216\left(x_{1}-x_{2}\right) x_{2}\left(x_{2}-\frac{1}{12}\right), & \mathbf{x} \in T_{2}, \\ 216\left(x_{1}-x_{3}\right) x_{3}\left(x_{3}-\frac{1}{12}\right), & \mathbf{x} \in T_{3}, \\ 216\left(x_{1}-x_{4}\right) x_{4}\left(x_{4}-\frac{1}{12}\right), & \mathbf{x} \in T_{4} .\end{cases}
$$

With $\mathbf{u}=\left(\begin{array}{lll}0 & v_{3,1} & 0\end{array}\right)^{\top}$ in (5) it would imply $\int_{T_{2}} x_{1} x_{2} p d \mathbf{x}=\int_{T_{4}} x_{1} x_{4} p d \mathbf{x}$. By symmetries, we would get

$$
\int_{T_{i}} x_{i} x_{j} p d \mathbf{x}=\int_{T_{i}} x_{i} x_{l} p d \mathbf{x}, \quad l \neq i \neq j .
$$

Letting $\mathbf{u}=\left(\begin{array}{lll}v_{3,1} & 0 & 0\end{array}\right)^{\top}$ in (5), by (12)-(13), it follows that

$$
\int_{T_{2}} x_{2}^{2} p d \mathbf{x}+\int_{T_{3}} x_{3}^{2} p d \mathbf{x}+2 \int_{T_{4}} x_{4}^{2} p d \mathbf{x}=2 \int_{T_{4}} x_{1} x_{4} p d \mathbf{x} .
$$

By (12), we have

$$
2 \int_{T_{1}} x_{1}^{2} p d \mathbf{x}=\int_{T_{4}} x_{i} x_{4} p d \mathbf{x}, \quad 1 \leq i \leq 3 .
$$

Adding (14) for $i=1,2,3$, we get

$$
6 \int_{T_{1}} x_{1}^{2} p d \mathbf{x}=\int_{T_{4}}\left(x_{1}+x_{2}+x_{3}\right) x_{4} p d \mathbf{x}=\int_{T_{4}}\left(1-x_{4}\right) x_{4} p d \mathbf{x} .
$$

Hence,

Therefore,

$$
7 \int_{T_{1}} x_{1}^{2} p d \mathbf{x}=\int_{T_{4}} x_{4} p d \mathbf{x}=0
$$

$$
\int_{T_{i}} x_{i}^{2} p d \mathbf{x}=\int_{T_{i}} x_{i} x_{j} p d \mathbf{x}=0, \quad 1 \leq i, j \leq 4 .
$$

Next we let $v_{3,2}$ be the nodal basis function at the other node on the edge ae: $(2 a+e) / 3$. This time, after letting $\mathbf{u}=\left(0 v_{3,2} 0\right)^{\top}$ in (5), it follows that $\int_{T_{i}} x_{j}^{2} p d \mathbf{x}=$ $7 \int_{T_{i}} x_{i} x_{j} p d \mathbf{x}=0$ for $i \neq j$. Finally, let $v_{3, f}$ be the nodal basis function at the node $(a+d+e) / 3$ inside a face triangle ade. Then $\mathbf{u}=\left(\begin{array}{lll}0 & v_{3, f} & 0\end{array}\right)^{\top}$ would lead us to $\int_{T_{2}} x_{1} x_{4} p d \mathbf{x}=13 \int_{T_{2}} x_{2}^{2} p d \mathbf{x}=0$. For the last step involving $v_{3, f}$, we can see more details in proving Theorem 4 as we will do it again for general degree $k \geq 3$.

Summarizing the three lemmas, we get, for $p$ satisfying conditions in Lemma 3,

$$
\int_{T_{i}} q p d \mathbf{x}=0 \text { for all } q \in P_{2, h_{0}, T_{i}}, \quad 1 \leq i \leq 4 .
$$

We thus proved special cases of the main theorem for $k=1,2$, and 3 . 


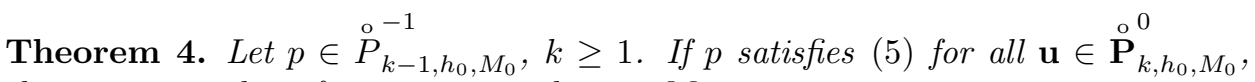
then $p \equiv 0$ on the reference macro-element $M_{0}$.

Proof. We use the principle of mathematical induction. We have proved the theorem for $k=0,1$ and 2. Assuming

$$
\int_{T_{i}} q p d \mathbf{x}=0 \text { for all degree } m \text { or less polynomials } q, \quad 1 \leq i \leq 4,
$$

holds for $m=k-2$, we need to prove (16) for $m=k-1$. By the degree- $k$ finite-element nodal basis function $v_{k, e}$ at the bary-center $e$,

$$
\left.v_{k, e}\right|_{T_{i}}=\frac{\prod_{j=1}^{k}\left(x_{i}-c_{j}\right)}{\prod_{j=1}^{k}\left(\frac{1}{4}-c_{j}\right)}, \quad 1 \leq i \leq 4, c_{j}=\frac{j-1}{4 k},
$$

we would show as in the lemmas that all values of $\int_{T_{i}} x_{i}^{k-1} p d \mathbf{x}$ are the same (cf. $(12))$. Then we work with the degree- $k(k \geq 2)$ finite-element nodal bases at nodes

$$
n_{l}=\frac{l}{k} a+\left(1-\frac{l}{k}\right) e=\left(\frac{k+3 l}{4 k}, \frac{k-l}{4 k}, \frac{k-l}{4 k}\right), \quad 0<l<k,
$$

inside edge ae (cf. Figure 1):

$$
v_{k, l}= \begin{cases}0 & \text { on } T_{1}, \\ C_{l} \prod_{j=1}^{k-l}\left(x_{2}-c_{j}\right) \prod_{j=1}^{l}\left(x_{1}-x_{2}-c_{j}\right) & \text { on } T_{2}, \\ C_{l} \prod_{j=1}^{k-l}\left(x_{3}-c_{j}\right) \prod_{j=1}^{l}\left(x_{1}-x_{3}-c_{j}\right) & \text { on } T_{3}, \\ C_{l} \prod_{j=1}^{k-l}\left(x_{4}-c_{j}\right) \prod_{j=1}^{l}\left(x_{1}-x_{4}-c_{j}\right) & \text { on } T_{4},\end{cases}
$$

where the $c_{j}$ are defined in (17) and the $C_{l}$ are constants defined properly such that $v_{k, l}$ assumes value 1 at the node $n_{l}$. Letting $\mathbf{u}=\left(\begin{array}{ll}0 & v_{k, 1}\end{array}\right)^{\top}$ in $(5)$, we get, by (16), that

$$
\begin{gathered}
-k C_{l} \int_{T_{2}} x_{2}^{k-1} p d \mathbf{x}+(k-1) C_{l} \int_{T_{2}} x_{1} x_{2}^{k-2} p d \mathbf{x} \\
=-k C_{l} \int_{T_{4}} x_{4}^{k-1} p d \mathbf{x}+(k-1) C_{l} \int_{T_{4}} x_{1} x_{4}^{k-2} p d \mathbf{x} \\
\Rightarrow \int_{T_{2}} x_{1} x_{2}^{k-2} p d \mathbf{x}=\int_{T_{4}} x_{1} x_{4}^{k-2} p d \mathbf{x} .
\end{gathered}
$$

Here and below, by mathematical induction (16), we only need to record the integrals of the product of $p$ and degree $(k-1)$ polynomial terms, since all the integrals with lower degree terms are zero by (16). By symmetries or by using nodal bases on the other three internal edges, it follows that all the values of $\int_{T_{i}} x_{j} x_{i}^{k-2} p d \mathbf{x}$ are the same. Now by letting $\mathbf{u}=\left(\begin{array}{lll}v_{k, 1} & 0 & 0\end{array}\right)^{\top}$ in (5), we get

$$
\begin{gathered}
\int_{T_{2}} x_{2}^{k-1} p d \mathbf{x}+\int_{T_{3}} x_{3}^{k-1} p d \mathbf{x}-(k-1) \int_{T_{4}}\left(x_{1}-x_{4}\right) x_{4}^{k-2} p d \mathbf{x}=0 \Rightarrow \\
(k+1) \int_{T_{2}} x_{2}^{k-1} p d \mathbf{x}=(k-1) \int_{T_{4}} x_{1} x_{4}^{k-2} p d \mathbf{x} .
\end{gathered}
$$


By symmetries and repeating the process after (11), i.e., adding the three equations

$$
\begin{aligned}
& (k+1) \int_{T_{2}} x_{2}^{k-1} p d \mathbf{x}=(k-1) \int_{T_{4}} x_{1} x_{4}^{k-2} p d \mathbf{x}, \\
& (k+1) \int_{T_{2}} x_{2}^{k-1} p d \mathbf{x}=(k-1) \int_{T_{4}} x_{2} x_{4}^{k-2} p d \mathbf{x}, \\
& (k+1) \int_{T_{2}} x_{2}^{k-1} p d \mathbf{x}=(k-1) \int_{T_{4}} x_{3} x_{4}^{k-2} p d \mathbf{x},
\end{aligned}
$$

we then get, as before, using $x_{4}=1-x_{1}-x_{2}-x_{3}$,

$$
(4 k+2) \int_{T_{i}} x_{i}^{k-1} p d \mathbf{x}=(k-1) \int_{T_{4}} x_{4}^{k-2} p d \mathbf{x}=0, \quad 1 \leq i \leq 4 .
$$

By (18) we proved that (note $l$ is only 0 and 1 !)

$$
\int_{T_{i}} x_{j}^{l} x_{i}^{k-1-l} p d \mathbf{x}=0, \quad 1 \leq i, j \leq 4,0 \leq l \leq 1
$$

Let $\mathbf{u}=\left(\begin{array}{lll}0 & v_{k, 2} & 0\end{array}\right)^{\top}$ in (5). It follows that, by the newly proved results (19),

$$
\int_{T_{2}} x_{1}^{2} x_{2}^{k-3} p d \mathbf{x}=\int_{T_{4}} x_{1}^{2} x_{4}^{k-3} p d \mathbf{x}
$$

and that all values of $\int_{T_{i}} x_{j}^{2} x_{i}^{k-3} p d \mathbf{x}$ are the same. Letting $\mathbf{u}=\left(\begin{array}{lll}v_{k, 2} & 0 & 0\end{array}\right)^{\top}$ in (5), we prove (19) for $l=2$. Repeatedly using $v_{k, l}$ for $l>2$, we show (19) for all other $l$ :

$$
\int_{T_{i}} x_{j}^{l} x_{i}^{k-1-l} p d \mathbf{x}=0, \quad 0 \leq l \leq k-1, \quad 1 \leq i, j \leq 4
$$

We next work on the cases with three variables such as $x_{1}^{i_{1}} x_{2}^{i_{2}} x_{3}^{i_{3}}$ where $i_{1}+i_{2}+$ $i_{3}=k-1$. We use the nodal basis functions involving three variables, i.e., not those associated with nodes on edges but on faces. At these face nodes

$$
n_{i, j}=\frac{i}{k} a+\frac{j}{k} d+\frac{k-i-j}{k} e, \quad i \geq 1, j \geq 1, k-i-j \geq 1,
$$

in the interior of triangle ade (see Figure 1), the degree- $k$ nodal bases are

$$
v_{k, i, j}=C_{i j} \begin{cases}\prod_{l=1}^{k-i-j}\left(x_{2}-c_{l}\right) \prod_{l=1}^{i}\left(x_{1}-x_{2}-c_{l}\right) \prod_{l=1}^{j}\left(x_{4}-x_{2}-c_{l}\right) & \text { on } T_{2}, \\ \prod_{l=1}^{k-i-j}\left(x_{3}-c_{l}\right) \prod_{l=1}^{i}\left(x_{1}-x_{3}-c_{l}\right) \prod_{l=1}^{j}\left(x_{4}-x_{3}-c_{l}\right) & \text { on } T_{3}, \\ 0 & \text { on } T_{4},\end{cases}
$$

where the $c_{l}$ are defined in (17) and the $C_{i j}$ are defined properly such that $v_{k, i, j}=1$ at the node $n_{i, j}$. Let the $\mathbf{u}$ in (5) be $\left(0 v_{k, 1,1} 0\right)^{\top}$. When we compute (5), we only need to watch the terms of three variables and of highest order, because of (16) and (20). This would easily lead to

$$
-(k-2) \int_{T_{2}} x_{1} x_{3} x_{2}^{k-3} p d \mathbf{x}=0 .
$$


By symmetries, it holds that

$$
\int_{T_{l}} x_{m} x_{n} x_{l}^{k-3} p d \mathbf{x}=0, \quad 1 \leq l \leq 4, m \neq n \neq l \neq m .
$$

In (5), letting $\mathbf{u}=\left(\begin{array}{lll}0 & v_{k, i, j} & 0\end{array}\right)^{\top}$ in an order of increasing $(i+j)$, we would show sequentially that

$$
\begin{aligned}
\int_{T_{l}} x_{m}^{i} x_{n}^{j} x_{l}^{k-3-i-j} p d \mathbf{x}=0, \quad 1 \leq i, j \leq k-2, \\
i+j \leq k-1, \quad 1 \leq l \leq 4, m \neq n \neq l \neq m .
\end{aligned}
$$

Therefore, combining (16) with $m=(k-2),(20)$ and (21), we get (16) again for $m=(k-1)$. The proof is complete.

If $p \in \stackrel{\circ}{P}_{k-1}$, then a linear combination of (16) leads to $\int_{M_{0}} p^{2}=0$ and $p \equiv 0$.

Let us make a remark on the main result of the paper, Theorem 4, which says that

$$
\operatorname{div} \stackrel{\circ}{\mathbf{P}}_{k, h_{0}, M_{0}}^{0}=\stackrel{\circ}{P}_{k-1, h_{0}, M_{0}}^{-1}
$$

on the reference macro-element $M_{0}$. The divergence of the space of polynomials of degree $k$ or less is exactly the space of polynomials of degree $(k-1)$ or less. When posting the boundary condition, say, for a domain consisting of one tetrahedron, we will lose a lot of degrees of freedom for the discrete velocity space, the space of polynomials of degree $k$ or less satisfying the zero boundary condition. To be exact, we lose all degrees of freedom if $k \leq 4$, and for $k>4$,

$$
\frac{(k+3)(k+2)(k+1)}{3}-\frac{(k-1)(k-2)(k-3)}{3} .
$$

Meanwhile, we lose only one degree of freedom for the discrete pressure space, the polynomials of degree $(k-1)$ or less having mean-value zero. The specially designed macro-element creates a lot of internal degrees of freedom for the discrete velocity space so that the match is perfect. This macro-element is made by the simplest grid on a single tetrahedron for the two spaces to match.

Theorem 5. Suppose that the solution of (1) satisfies $\vec{\psi} \in H^{r}(\Omega)^{3}, r \geq 1$, and $\tau \in H^{s}(\Omega), s \geq 0$, and let $\left(\mathbf{u}_{h}, p_{h}\right) \in \stackrel{\circ}{\mathbf{P}}_{k, h, \Omega}^{0} \times \stackrel{\circ}{P}_{k-1, h, \Omega}^{-1}$ be the solution of (2). If $k \geq 3$, the following error estimate holds:

$$
\left|\vec{\psi}-\mathbf{u}_{h}\right|_{1}+\left\|\tau-p_{h}\right\|_{0} \leq C\left(h^{q_{1}-1}\|\vec{\psi}\|_{q_{1}}+h^{q_{2}}\|\tau\|_{q_{2}}\right),
$$

where $q_{1}=\min \{r, k+1\}$ and $q_{2}=\min \{s, k\}$.

Proof. For any $M \in \mathcal{M}_{h}$ and any $p \in N_{M}$ (defined in (4)), let $\bar{p}=\int_{M} p$. We then have $p-\bar{p} \in \stackrel{\circ}{P}_{k-1, h, M}^{-1}$ and Theorem 4 shows that $p-\bar{p} \equiv 0$ on $M$ (after an affine mapping) for all $k \geq 0$, which means $p$ is a constant function $\bar{p}$ on $M$. Therefore $\operatorname{dim}\left(N_{M}\right)=1$ and (4) holds, which ensures (3) when $k \geq 3$ as proved by Stenberg in [1]. The approximation result follows the stability condition (3) (cf., e.g. [2]).

We remark that the condition $k \geq 3$ in Theorem 5 is sharp in some sense. This can be seen by considering a domain consisting of two tetrahedra. We then subdivide each of the two tetrahedra to get eight macro-element type subtetrahedra. On such a mesh, the dimension of the discrete velocity space is smaller than that 
of the discrete pressure space: $\operatorname{dim}\left({\stackrel{\circ}{\mathbf{P}^{0}}}_{2, h, \Omega}^{0}\right)=3 \times 2 \times 5=30$ and $\operatorname{dim}\left(\stackrel{\circ}{P}_{1, h, \Omega}^{-1}\right)=$ $2 \times 4 \times 4-1=31$. This example shows the sharpness of the analysis in Stenberg 11 too. The $k \geq 3$ is required in the Stenberg framework, which ensures at least one internal node for the discrete velocity on the interface between any two 3D macro-elements. Stenberg's macro-element theory directly implies that the $\mathbf{P}_{3}-P_{0}$ mixed element is stable on tetrahedral grids. But, of course, one would not use such a method since it wastes "two orders" of computation. In other words, the stability of $3 \mathrm{D} \mathbf{P}_{3}-P_{0}$ elements implies the stability of our macro $\mathbf{P}_{3}-P_{2}$, or higher degree, elements.

\section{Numerical tests}

We will verify Theorem 4 by two numerical examples in this section. In the first case, the domain is the unit right tetrahedron at the origin (reference macro-element $M_{0}$ ). We subdivide the domain into four tetrahedra which make a macro-element. We compute the singular values of the matrix $A=\left(a_{i j}\right)$ where $a_{i j}$ are the values of the divergence of the Lagrange nodal basis for $\stackrel{\circ}{\mathbf{P}}_{k, h, \Omega}^{0}$ at nodes of the Lagrange basis for $\stackrel{\circ}{P}_{k-1, h, \Omega}^{-1}$. In Table 1 , we list the maximal and minimal singular values of $A$. Since $A$ is a full-rank matrix, it implies that the image of divergence of $\stackrel{\circ}{\mathbf{P}}_{k}$ is $\stackrel{\circ}{P}_{k-1}$ and that Theorem 4 holds in this testing case.

In the second test, the domain is the unit cube with initial mesh consisting of six tetrahedra. We get the macro-element mesh $\mathcal{T}_{h}$ by subdividing the six tetrahedra into 24 tetrahedra. In Table 2, we list the maximal and minimal singular values of $A$. The data show that the condition $k \geq 3$ is necessary in Theorem 5 .

TABLE 1. Singular values in the case of one tetrahedron domain

\begin{tabular}{|c|r|r||r|r|r|}
\hline \multirow{2}{*}{$\begin{array}{c}\text { Degree } \\
k\end{array}$} & \multicolumn{2}{|c||}{ Singular Values } & $\operatorname{dim}\left(\stackrel{\circ}{\mathbf{P}}_{k}\right)$ & $\operatorname{dim}\left(\stackrel{\circ}{P}_{k-1}^{-1}\right)$ & difference \\
\cline { 2 - 4 } & Minimal & Maximal & & 3 & 0 \\
\hline 1 & 1.0000 & 2.0000 & 3 & 15 & 0 \\
\hline 2 & 1.1856 & 10.5279 & 15 & 39 & 6 \\
\hline 3 & 1.2365 & 25.3072 & 45 & 79 & 26 \\
\hline 4 & 1.0176 & 51.9827 & 105 & 139 & 68 \\
\hline 5 & 0.7936 & 101.0454 & 207 & 223 & 140 \\
\hline 6 & 0.7903 & 193.8626 & 363 & 335 & 250 \\
\hline 7 & 0.8074 & 372.5908 & 585 & 479 & 406 \\
\hline 8 & 0.8178 & 720.7702 & 885 & & \\
\hline
\end{tabular}

TABLE 2. Singular values in the case of one cube domain

\begin{tabular}{|c|r|r||r|r|r|}
\hline \multirow{2}{*}{$\begin{array}{c}\text { Degree } \\
k\end{array}$} & \multicolumn{2}{|c||}{ Singular Values } & $\operatorname{dim}\left(\stackrel{\circ}{\mathbf{P}}_{k}\right)$ & $\operatorname{dim}\left(\stackrel{\circ}{P}_{k-1}^{-1}\right)$ & difference \\
\cline { 2 - 3 } & Minimal & Maximal & & & \\
\hline 1 & - & - & 18 & 23 & -5 \\
\hline 2 & - & - & 93 & 95 & -2 \\
\hline 3 & 2.7663 & 94.9330 & 294 & 239 & 55 \\
\hline 4 & 2.3348 & 203.4540 & 693 & 479 & 214 \\
\hline 5 & 2.1847 & 379.1579 & 1362 & 839 & 523 \\
\hline
\end{tabular}



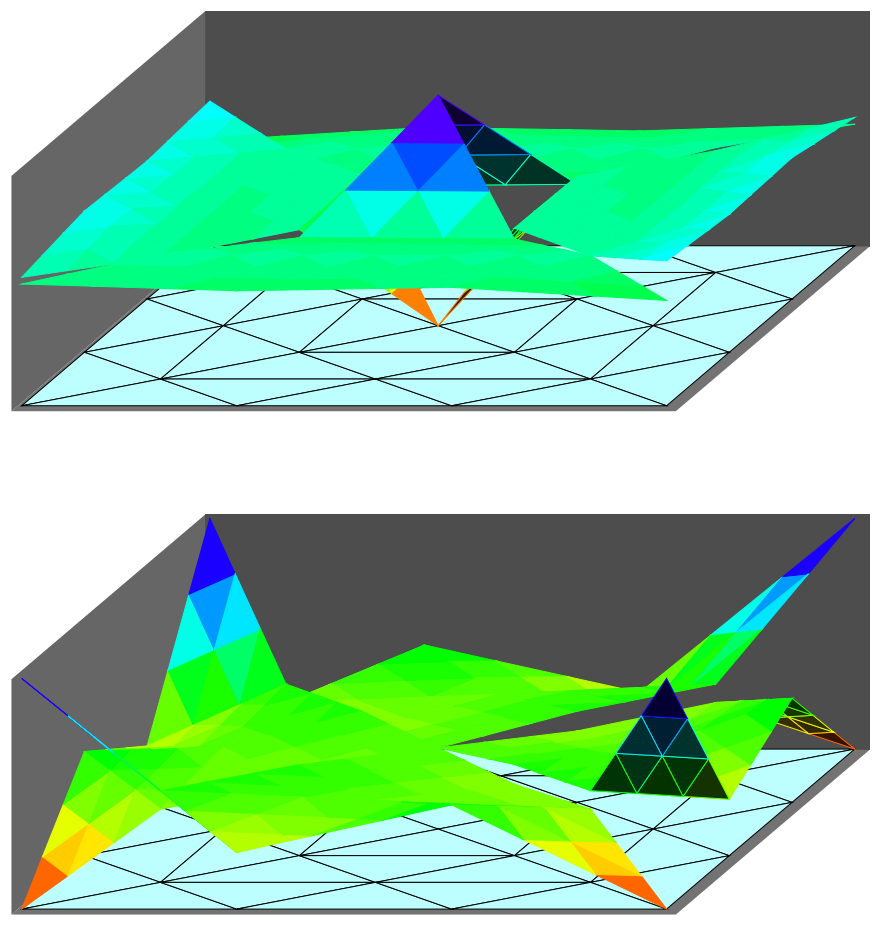

Figure 2. The errors for the discrete pressure $\left(\mathbf{P}_{4}-P_{3}\right)$ when a singular vertex presents. The discrete linear system is solved by the Gaussian elimination (top) or by the iterated penalty method (bottom, at a different scaling, where the maximal nodal error is $1 / 20$ of that on the top).

Finally, we make a few additional comments on singular vertices. For each additional singular vertex in $2 \mathrm{D}$, the space of discrete divergence-free velocity has one more dimension. Therefore, the finite element space for velocity would be less "locking" and "singular vertices" are good in some sense. However, we should point out that this might be true only for lower-degree finite element spaces. For example, the degrees of freedom of divergence-free velocity in some $\mathbf{P}_{1}-P_{0}$ mixed elements are mainly provided by singular vertices in [6]. Furthermore, even "nearly singular vertices" are not necessarily bad since they could provide an additional approximation for the unknown velocity, depending on what and how iterative methods are applied to the discrete linear system. In Figure 2, we plot the errors of computed pressure when solving Stokes equations on the unit square, where the velocity $\vec{\phi}=\operatorname{curl} g$ and the pressure $\tau=-\Delta g$, for $g=\left(x-x^{2}\right)^{2}\left(y-y^{2}\right)^{2}$, by $\mathbf{P}_{4}-P_{3}$ mixed elements. The error for discrete velocity is typical of the optimal order. We used both the Gaussian elimination and an iterated penalty method [10]. When a singular vertex (at the center in Figure 2) or a nearly singular vertex is present, the Gaussian elimination method (without filtering out the extra degree of freedom in discrete pressure) would produce a big nodal error there for the discrete pressure, 
because the linear system is singular (within the round-off error) or nearly singular. However, for the iterated penalty method or Uzawa, or incomplete Uzawa, or most other iterative methods, the discrete pressure solution from the iteration does not have any unusual error near the singular vertex, shown in Figure 2. This is because most iterative methods would smartly limit the iterates for pressure within the proper subspace or not too far off the subspace (see [7].) The conclusion we would draw is still that we should avoid singular/nearly singular vertices if possible, for high-degree mixed elements.

\section{REFERENCES}

1. D. N. Arnold, F. Brezzi and M. Fortin, A stable finite element for the Stokes equations, Calcolo 21 (1984), 337 - 344. MR86m:65136

2. F. Brezzi and M. Fortin, Mixed and hybrid finite element methods, Springer-Verlag, Berlin, 1991. MR92d:65187

3. P. G. Ciarlet, The Finite Element Method for Elliptic Problems, North-Holland, Amsterdam, New York, Oxford, 1978. MR58:25001

4. V. Girault and P. A. Raviart, Finite Element Methods for Navier-Stokes Equations, SpringerVerlag, New York-Heidelberg-Berlin, 1986. MR88b:65129

5. J. Qin, On the Convergence of Some Low Order Mixed Finite Elements for Incompressible Fluids, Ph. Dissertation, Department of Mathematics, Pennsylvania State University, 1994.

6. J. Qin and S. Zhang, Stability and approximability of the $\mathcal{P}_{1}-\mathcal{P}_{0}$ element for Stokes equations, preprint.

7. J. Qin and S. Zhang, A general convergence theory on preconditioned linear iterations for saddle point problems, unpublished manuscript.

8. L. R. Scott and M. Vogelius, Norm estimates for a maximal right inverse of the divergence operator in spaces of piecewise polynomials, Math. Modelling Numer. Anal. 19 (1985), $111-$ 143. MR87i:65190

9. L. R. Scott and S. Zhang, Finite-element interpolation of non-smooth functions satisfying boundary conditions, Math. Comp. 54 (1990), 483 - 493. MR90j:65021

10. L. R. Scott and S. Zhang, Multilevel iterated penalty method for mixed elements, The Proceedings for the Ninth International Conference on Domain Decomposition Methods, Bergen, 1998, pp. 133-139.

11. R. Stenberg, Analysis of mixed finite element methods for the Stokes problem: A unified approach, Math. Comp. 42 (1984), 9 - 23. MR.84k:76014

12. R. Stenberg, On some three-dimensional finite elements for incompressible media, Comput. Methods Appl. Mech. Engrg. 63 (1987), 261 - 269. MR88i:73050

13. S. Zhang, Multi-level Iterative Techniques, Ph. Dissertation, Department of Mathematics, Pennsylvania State University, 1988.

14. S. Zhang, Successive subdivisions of tetrahedra and multigrid methods on tetrahedral meshes, Houston J. of Math. (1995), 541 - 556. MR96f:65183

15. S. Zhang, An optimal order multigrid method for biharmonic, $C^{1}$ finite-element equations, Numer. Math. 56 (1989), 613 - 624. MR90j:65135

16. S. Zhang, Optimal order non-nested multigrid methods for solving finite element equations I: on quasiuniform meshes, Math. Comp. 55 (1990), 23 - 36. MR91g:65268

Department of Mathematical Sciences, University of Delaware, Newark, Delaware 19716

E-mail address: szhang@math.udel.edu 